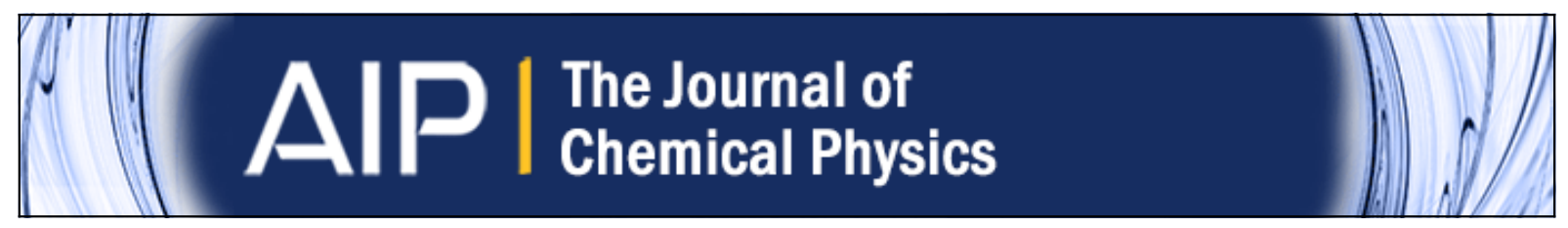

Contact line motion in confined liquid-gas systems: Slip versus phase transition

Xinpeng Xu and Tiezheng Qian

Citation: The Journal of Chemical Physics 133, 204704 (2010); doi: 10.1063/1.3506886

View online: http://dx.doi.org/10.1063/1.3506886

View Table of Contents: http://scitation.aip.org/content/aip/journal/jcp/133/20?ver=pdfcov

Published by the AIP Publishing

Articles you may be interested in

A slip model for rarefied gas flows above a moving surface with mass transfer

J. Appl. Phys. 116, 054503 (2014); 10.1063/1.4891840

Numerical study of drop motion on a surface with stepwise wettability gradient and contact angle hysteresis Phys. Fluids 26, 062101 (2014); 10.1063/1.4880656

Slip effects at the vapor-liquid boundary

AIP Conf. Proc. 1501, 903 (2012); 10.1063/1.4769638

On the effects of liquid-gas interfacial shear on slip flow through a parallel-plate channel with superhydrophobic grooved walls

Phys. Fluids 22, 102002 (2010); 10.1063/1.3493641

Numerical analysis of thermal-slip and diffusion-slip flows of a binary mixture of hard-sphere molecular gases Phys. Fluids 15, 3745 (2003); 10.1063/1.1624075

How can you REACH $100 \%$ of researchers at the Top 100 Physical Sciences Universities?

With The Journal of Chemical Physics.

AIP $\left.\right|_{\text {Chemical Physics }} ^{\text {The Journal of }}$

THERE'S POWER IN NUMBERS. Reach the world with AIP Publishing.

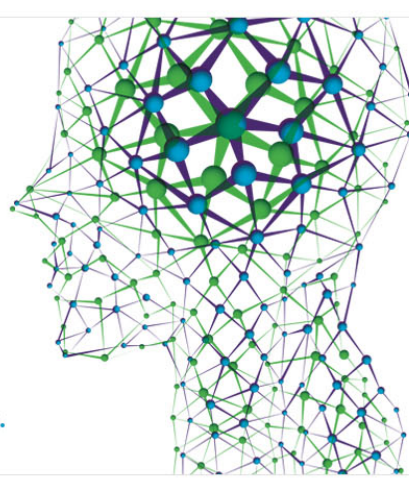




\title{
Contact line motion in confined liquid-gas systems: Slip versus phase transition
}

\author{
Xinpeng $\mathrm{Xu}^{1}$ and Tiezheng Qian ${ }^{2, a)}$ \\ ${ }^{1}$ Nano Science and Technology (NSNT) Program, Hong Kong University of Science and Technology, \\ Clear Water Bay, Kowloon, Hong Kong \\ ${ }^{2}$ Department of Mathematics and KAUST-HKUST Micro/Nanofluidics Joint Laboratory, Hong Kong University \\ of Science and Technology, Clear Water Bay, Kowloon, Hong Kong
}

(Received 11 August 2010; accepted 8 October 2010; published online 30 November 2010)

\begin{abstract}
In two-phase flows, the interface intervening between the two fluid phases intersects the solid wall at the contact line. A classical problem in continuum fluid mechanics is the incompatibility between the moving contact line and the no-slip boundary condition, as the latter leads to a nonintegrable stress singularity. Recently, various diffuse-interface models have been proposed to explain the contact line motion using mechanisms missing from the sharp-interface treatments in fluid mechanics. In one-component two-phase (liquid-gas) systems, the contact line can move through the mass transport across the interface while in two-component (binary) fluids, the contact line can move through diffusive transport across the interface. While these mechanisms alone suffice to remove the stress singularity, the role of fluid slip at solid surface needs to be taken into account as well. In this paper, we apply the diffuse-interface modeling to the study of contact line motion in one-component liquid-gas systems, with the fluid slip fully taken into account. The dynamic van der Waals theory has been presented for one-component fluids, capable of describing the two-phase hydrodynamics involving the liquid-gas transition [A. Onuki, Phys. Rev. E 75, 036304 (2007)]. This theory assumes the local equilibrium condition at the solid surface for density and also the no-slip boundary condition for velocity. We use its hydrodynamic equations to describe the continuum hydrodynamics in the bulk region and derive the more general boundary conditions by introducing additional dissipative processes at the fluid-solid interface. The positive definiteness of entropy production rate is the guiding principle of our derivation. Numerical simulations based on a finite-difference algorithm have been carried out to investigate the dynamic effects of the newly derived boundary conditions, showing that the contact line can move through both phase transition and slip, with their relative contributions determined by a competition between the two coexisting mechanisms in terms of entropy production. At temperatures very close to the critical temperature, the phase transition is the dominant mechanism, for the liquid-gas interface is wide and the density ratio is close to 1 . At low temperatures, the slip effect shows up as the slip length is gradually increased. The observed competition can be interpreted by the Onsager principle of minimum entropy production. () 2010 American Institute of Physics. [doi:10.1063/1.3506886]
\end{abstract}

\section{INTRODUCTION}

What happens at the fluid-solid interface has been a classical problem that is particularly relevant to the study of multiphase fluid flows. The moving contact line (MCL) problem has been an unsolved classical problem in continuum hydrodynamics for decades. The contact line denotes the intersection of the fluid-fluid interface with a solid wall. When one fluid displaces the other, the contact line moves along the wall. It has been well known that the MCL is incompatible with the no-slip boundary condition ${ }^{1}$ - the latter leads to a nonintegrable singularity in viscous dissipation. ${ }^{2-5}$ As shown by Dussan and Davis, ${ }^{3}$ under usual hydrodynamic assumptions, namely, the incompressible Newtonian fluids, smooth rigid solid walls, impenetrable fluid-fluid interface, and no-slip boundary condition, there is a velocity discontinuity at the

\footnotetext{
a) Author to whom correspondence should be addressed. Electronic mail: maqian@ust.hk.
}

MCL, and the tangential force exerted by the fluids on the solid bounding surface in the vicinity of the MCL is infinite.

The heart of the MCL problem lies in the boundary condition(s) at the fluid-solid interface. In particular, molecular dynamics (MD) simulations showed that fluid slip indeed occurs at the MCL. ${ }^{6,7}$ Numerous models had been proposed over the years, ${ }^{8-17}$ but none was able to give a quantitative account of the fluid slip measured in MD simulations. In fact, there has been a lasting debate over the boundary conditions for a fluid flowing past a solid surface. In recent years, the Newtonian flows in confined geometries have received much attention, and numerous research efforts have shown that fluid slips at the solid boundary in many circumstances. ${ }^{18,19}$ The magnitude of slip is characterized by a quantity of length unit, referred to as the slip length. For a simple shear flow parallel to the surface, it is the distance below the surface at which the fluid velocity extrapolates to zero (no slip). The slip length usually ranges from a few angstroms to a few nanometers. This explains why the no-slip boundary 
condition works perfectly well in macroscopic flows, and also why an accurate account of slip is needed for flows of micro/nanoscale. While single-phase flows require the slip effect to be taken into account only at small length scales, the contact line motion in two-phase flows is incompatible with the no-slip boundary condition even if the system dimension (e.g., the size of a droplet spreading at a solid surface) is on macroscopic length scale.

MD simulations have proven to be instrumental in investigating the fluid dynamics in the molecular scale vicinity of the MCL in two-component fluids. Through analysis of extensive MD data, we found that the fluid slip measured in nanoscale MD simulations is governed by the generalized Navier boundary condition (GNBC) ${ }^{20}$ The GNBC states that the relative slip velocity between the fluid and the solid wall is proportional to the total tangential stress-the sum of the viscous stress and the uncompensated Young stress; the latter arises from the deviation of the fluid-fluid interface from its static configuration. By combining the GNBC with the Cahn-Hilliard hydrodynamic formulation for immiscible two-phase flows, ${ }^{14,15,17,20}$ we have obtained a continuum model for MCL hydrodynamics. ${ }^{20}$ Its numerical implementation has produced continuum solutions in quantitative agreement with MD simulation results. ${ }^{20,21}$ Recently, it has been shown $^{22,23}$ that the GNBC can be derived in a variational approach based on the Onsager principle of minimum energy dissipation. ${ }^{24,25}$ We would like to point out that in all the other studies based on diffuse-interface modeling for binary mixtures, ${ }^{14,15,17}$ the no-slip boundary condition is kept and the nonintegrable stress singularity is removed by introducing diffusive transport through the fluid-fluid interface. By combining the GNBC with the diffuse-interface formulation, our continuum model allows the coexistence of slip and diffusion, with their competition determined by the relative magnitudes of relevant parameters. ${ }^{20,22}$ For the systems simulated in our MD study, it is the slip that dominates. A recent study by Ren and $\mathrm{E},{ }^{26}$ which focused on a sharp interface model, also demonstrated that the stress singularity is regularized by the existence of a slip region and the Young stress is dominant in the contact line region.

MCL also exists in one-component two-phase fluids, i.e., liquid-gas systems. Compared to the immiscible binary fluids, the liquid-gas systems allow the contact line to move solely through phase transition (evaporation/condensation), and mathematically, there is no stress singularity due to the no-slip boundary condition applied at the solid surface. There have been several studies of the MCL in liquid-gas systems by the use of diffuse-interface models subject to the no-slip boundary condition. ${ }^{13,16,27-30}$

The purpose of this paper is to apply the diffuse-interface modeling $^{31}$ to the study of contact line motion in onecomponent liquid-gas systems, with the fluid slipping at the solid surface fully taken into account. These systems are distinguished from the binary fluids by the fact that phase transition, signaled by strong heat transfer and mass transport across the interface, is naturally involved in contact line motion. The dynamic van der Waals theory ${ }^{30}$ has been presented for one-component fluids, capable of describing the two-phase hydrodynamics involving the liquid-gas transition in inhomogeneous temperature. We will make use of its hydrodynamic equations, which contain the reversible stress tensor arising from the density gradient, to describe the continuum hydrodynamics in the bulk region. As for the boundary conditions at solid surface, the fluid slip effect has to be taken into account. We will derive the general boundary conditions for the dynamic van der Waals theory and then carry out numerical simulations for two-phase Couette flows confined between two planar solid walls. By taking into account the dissipative processes (e.g., slip) at the fluid-solid interface, our study will provide a more complete understanding of the MCL in liquid-gas systems. It will lead to a quantitatively more accurate description for the flow phenomena involved in confined geometries and wetting dynamics, ${ }^{5}$ which are important to many industrial processes.

The paper is organized as follows. In Sec. II, the dynamic van der Waals theory is reviewed first. The boundary conditions are then derived by introducing additional dissipative processes at the fluid-solid interface. The details of our numerical simulations are given in Sec. III. The numerical results are presented in Sec. IV, with a theoretical interpretation based on the Onsager principle of minimum energy dissipation (entropy production). It is shown that the contact line can move through both phase transition and slip, with their relative contributions determined by a competition between the two coexisting mechanisms. At temperatures very close to the critical temperature, the phase transition is dominant, for the liquid-gas interface is wide and the density ratio is close to one. At low temperatures, the slip effect shows up with the increasing slip length. The paper is concluded in Sec. V with a few remarks.

\section{THEORY}

In the first part of this section, there is a brief review of the dynamic van der Waals theory. ${ }^{30}$ This theory assumes the local equilibrium condition at the solid surface for density and also the no-slip boundary condition for velocity. In the second part, these two conditions are relaxed by introducing additional dissipative processes at the fluid-solid interface. The positive definiteness of entropy production rate will be the guiding principle for our derivation of the general boundary conditions.

\section{A. Dynamic van der Waals theory}

For a one-component system, the density is the order parameter that distinguishes the bulk fluids (liquid and gas) and the intervening interface. For monatomic molecules, the Helmholtz free energy density $f(n, T)$ as a function of the number density $n$ and the temperature $T$ is given by

$f(n, T)=n k_{B} T\left[\ln \left(\lambda_{\mathrm{th}}^{3} n\right)-1-\ln \left(1-v_{0} n\right)\right]-\varepsilon v_{0} n^{2}$,

where $k_{B}$ is the Boltzmann constant, $\lambda_{\text {th }}=\hbar\left(2 \pi / m k_{B} T\right)^{1 / 2}$ is the thermal de Broglie length with $m$ being the molecular mass, $v_{0}$ is the molecular volume, and $\varepsilon$ is the strength of attractive interaction. From $f(n, T)$, the internal energy density $e$, the entropy $s$ per molecule, and the pressure $p$ can be 
derived as functions of $n$ and $T$ by the use of thermodynamic relations:

$$
\begin{aligned}
& e(n, T)=\frac{3 n k_{B} T}{2-\varepsilon v_{0} n^{2}}, \\
& s(n, T)=-k_{B} \ln \left[\frac{\lambda_{\mathrm{th}}^{3} n}{\left(1-v_{0} n\right)}\right]+\frac{5 k_{B}}{2}, \\
& p(n, T)=\frac{n k_{B} T}{\left(1-v_{0} n\right)-\varepsilon v_{0} n^{2}},
\end{aligned}
$$

The van der Waals theory is generalized by including gradient contributions to the internal energy and the entropy. ${ }^{30}$ The internal energy density becomes

$$
\hat{e}=e+\frac{K(n)}{2}|\nabla n|^{2},
$$

while the entropy density becomes

$$
\hat{S}=n s-\frac{C(n)}{2}|\nabla n|^{2},
$$

with an increase of the energy and a decrease of the entropy due to the inhomogeneity of $n$. Maximizing the entropy $S_{b}=\int d \mathbf{r} \hat{S}$ for fixed particle number $N=\int d \mathbf{r} n$ and fixed internal energy $E_{b}=\int d \mathbf{r} \hat{e}$ (with respect to $e$ and $n$ as in a microcanonical ensemble) yields the bulk equilibrium conditions: (i) the homogeneity of temperature $T$ and (ii) the homogeneity of the generalized chemical potential

$$
\hat{\mu}=\mu+\frac{M_{n}}{2}|\nabla n|^{2}-T \nabla \cdot\left(\frac{M}{T} \nabla n\right),
$$

with $\mu=(e+p) / n-T s$ being the usual chemical potential in a homogeneous system, $M(n, T)=K(n)+C(n) T$, and $M_{n}=(\partial M / \partial n)_{T}$. When $T$ is homogeneous, it is useful to introduce the bulk Helmholtz free energy $F_{b}=E_{b}-T S_{b}$, given by

$$
F_{b}=\int d \mathbf{r}\left[f(n, T)+\frac{M(n, T)}{2}|\nabla n|^{2}\right],
$$

with $f(n, T)=e-T n s$. Minimizing $F_{b}$ with respect to $n$ yields the homogeneity of $\hat{\mu}$ again. To include the boundary effects at the surface of fluid container, the surface energy and entropy, of the forms $E_{s}=\int d A e_{s}(n)$ and $S_{s}=\int d A \sigma_{s}(n)$, respectively, are introduced, with the area densities $e_{s}$ and $\sigma_{s}$ only depending on $n$ at the surface. For homogeneous temperature $T$, the surface Helmholtz free energy $F_{s}=E_{s}-T S_{s}$, which is given by $F_{s}=\int d A f_{s}(n, T)$ with $f_{s}(n, T)=e_{s}-T \sigma_{s}$, is also introduced. Minimizing the total Helmholtz free energy $F_{\text {tot }}=F_{b}+F_{s}$ with respect to $n$ at the surface yields the surface equilibrium condition:

$$
L=0 \text {, }
$$

in which $L$ is a quantity defined at the solid surface by $L=M \nabla_{\gamma} n+\left(\partial f_{s} / \partial n\right)_{T}$, with $\gamma$ denoting the outward normal direction at the solid surface.

The hydrodynamic equations are given by

$$
\begin{aligned}
& \frac{\partial n}{\partial t}=-\nabla \cdot(n \mathbf{v}), \\
& \frac{\partial(\rho \mathbf{v})}{\partial t}+\nabla \cdot(\rho \mathbf{v v})=\nabla \cdot(-\overleftrightarrow{\Pi}+\stackrel{\leftrightarrow}{\sigma})-\rho g \hat{\mathbf{z}},
\end{aligned}
$$

$$
\frac{\partial \hat{e}}{\partial t}+\nabla \cdot(\hat{e} \mathbf{v})=-\overleftrightarrow{\Pi}: \nabla \mathbf{v}+\dot{\varepsilon}_{v}+\nabla \cdot(\lambda \nabla T),
$$

for the number density $n$, momentum density $\rho \mathbf{v}$, and internal energy density $\hat{e}$, respectively. Here $\rho=m n$ is the mass density, $g$ is the gravitational acceleration in the direction of $-\hat{\mathbf{z}},-\stackrel{\leftrightarrow}{\Pi}$ is the reversible stress tensor containing the gradient contributions, ${ }^{30} \stackrel{\leftrightarrow}{\sigma}$ is the viscous stress tensor, $\lambda$ is the thermal conductivity (with $-\lambda \nabla T$ being the heat current density), and $\dot{\varepsilon}_{v}$ is the rate of viscous heat production. From the expressions

$$
\begin{aligned}
& \Pi_{i j}=(n \hat{\mu}-\hat{e}+T \hat{S}) \delta_{i j}+M\left(\nabla_{i} n\right)\left(\nabla_{j} n\right), \\
& \sigma_{i j}=\eta\left(\nabla_{i} v_{j}+\nabla_{j} v_{i}\right)+(\zeta-2 \eta / 3) \delta_{i j} \nabla \cdot \mathbf{v},
\end{aligned}
$$

with $\eta$ and $\zeta$ denoting the shear and bulk viscosities, respectively, and

$$
\begin{aligned}
\dot{\varepsilon}_{v}=\overleftrightarrow{\sigma}: \nabla \mathbf{v}= & \frac{\eta}{2} \sum_{i j}\left(\nabla_{i} v_{j}+\nabla_{j} v_{i}-\frac{2}{3} \delta_{i j} \nabla \cdot \mathbf{v}\right)^{2} \\
& +\zeta(\nabla \cdot \mathbf{v})^{2} \geq 0
\end{aligned}
$$

the entropy density is found to satisfy

$$
\begin{aligned}
\frac{\partial}{\partial t} \hat{S}+\nabla \cdot(\hat{S} \mathbf{v})= & -\nabla \cdot\left[\frac{M \nabla n}{T}\left(\frac{\partial n}{\partial t}+\mathbf{v} \cdot \nabla n\right)-\frac{\lambda \nabla T}{T}\right] \\
& +\frac{\dot{\varepsilon}_{v}+\dot{\varepsilon}_{\theta}}{T},
\end{aligned}
$$

in which $\dot{\varepsilon}_{\theta}=\lambda(\nabla T)^{2} / T \geq 0$ is the rate of thermal heat production and $\left(\dot{\varepsilon}_{v}+\dot{\varepsilon}_{\theta}\right) / T$ is the rate of entropy production per unit volume in the bulk. The additional entropy flux $M \nabla n(\partial n / \partial t+\mathbf{v} \cdot \nabla n) / T$ is reversible and plays an essential role in our derivation of the relevant boundary conditions. Under the surface equilibrium condition $M \nabla_{\gamma} n+\left(\partial f_{s} / \partial n\right)_{T}$ $=0$ and the no-slip boundary condition, the rate of change of the total entropy $S_{\mathrm{tot}}=S_{b}+S_{s}$ is given by

$\frac{d S_{\text {tot }}}{d t}=\int d \mathbf{r} \frac{\dot{\varepsilon}_{v}+\dot{\varepsilon}_{\theta}}{T}+\int d A \frac{1}{T}\left(\lambda \nabla_{\gamma} T+\frac{\partial e_{s}}{\partial t}\right)$,

with $\partial e_{s} / \partial t=\left(\partial e_{s} / \partial n\right)(\partial n / \partial t) .{ }^{30}$ Additional rates of entropy production are expected to add to $d S_{\text {tot }} / d t$ if boundary conditions different from the above are used at the solid surface. This is to allow the density distribution to deviate from the local equilibrium and also to allow the fluid to slip.

\section{B. Rate of entropy production and boundary conditions}

The rate of change of the total entropy, $d S_{\text {tot }} / d t$ $=d S_{b} / d t+d S_{s} / d t$, is given by

$$
\begin{aligned}
\frac{d S_{\text {tot }}}{d t}= & \int d \mathbf{r} \frac{\dot{\varepsilon}_{v}+\dot{\varepsilon}_{\theta}}{T}+\int d A \frac{\lambda \nabla_{\gamma} T}{T}+\int d A\left[-\frac{M \nabla_{\gamma} n}{T}\right. \\
& \left.\times\left(\frac{\partial n}{\partial t}+v_{\tau} \nabla_{\tau} n\right)\right]+\int d A \frac{\partial \sigma_{s}}{\partial n} \frac{\partial n}{\partial t},
\end{aligned}
$$

in which the first three terms on the right-hand side are from the rate of change of the bulk entropy $d S_{b} / d t$ while the last term is from the rate of change of the surface entropy $d S_{s} / d t$. Here $d S_{b} / d t$ can be derived from Eq. (2.16) using $v_{\gamma}=0$ and $\mathbf{v} \cdot \nabla n=v_{\tau} \nabla_{\tau} n$ at the solid surface, with $\tau$ denoting the 
direction tangent to the solid surface (for two-dimensional flows). It is noted that the sum of the last two terms on the right-hand side of Eq. (2.18) can be reduced to a more instructive form:

$$
\begin{aligned}
& \int d A\left[-\frac{M \nabla_{\gamma} n}{T}\left(\frac{\partial n}{\partial t}+v_{\tau} \nabla_{\tau} n\right)+\frac{\partial \sigma_{s}}{\partial n} \frac{\partial n}{\partial t}\right] \\
& =\int d A\left\{\frac{1}{T}\left[(-\Pi)_{\gamma \tau} v_{\tau}-L \frac{\partial n}{\partial t}\right]+\frac{1}{T} \frac{\partial e_{s}}{\partial t}\right\},
\end{aligned}
$$

where $L=M \nabla_{\gamma} n+\left(\partial f_{s} / \partial n\right)_{T}$ has been defined in Sec. II A, with $f_{s}(n, T)=e_{s}-T \sigma_{s}$ being the area density of the surface Helmholtz free energy, and $(-\Pi)_{\gamma \tau} v_{\tau}-L \partial n / \partial t$ can be rewritten as

$$
\begin{aligned}
(-\Pi)_{\gamma \tau} v_{\tau}-L \frac{\partial n}{\partial t}= & {\left[(-\Pi)_{\gamma \tau}+\sigma_{\gamma \tau}\right] v_{\tau}+\left(L \nabla_{\tau} n-\sigma_{\gamma \tau}\right) v_{\tau} } \\
& -L\left(\frac{\partial n}{\partial t}+v_{\tau} \nabla_{\tau} n\right)
\end{aligned}
$$

Here, $v_{\tau}$ is the tangential velocity of fluid at surface, i.e., the slip velocity, $\left[(-\Pi)_{\gamma \tau}+\sigma_{\gamma \tau}\right] v_{\tau}$ is the rate of work per unit area done by the surface layer (a boundary fluid layer of thickness $0^{+}$) on the interior fluid, $L \nabla_{\tau} n-\sigma_{\gamma \tau}$ is the tangential force per unit area by the interior fluid on the surface layer due to (i) the liquid-gas interfacial tension (for $\left.M \nabla_{\gamma} n \nabla_{\tau} n=-(-\Pi)_{\gamma \tau}\right)$, (ii) the tangential variation of the surface free energy density (for $\left(\partial f_{s} / \partial n\right)_{T} \nabla_{\tau} n=\nabla_{\tau} f_{s}$ ), and (iii) the shear viscosity (for $-\sigma_{\gamma \tau}$ ), and $L$ is a measure of the deviation from the surface equilibrium. In order to derive the general boundary conditions at solid surface, we note that the rate of change of the total entropy can be written as

$$
\begin{aligned}
\frac{d S_{\text {tot }}}{d t}= & \int d \mathbf{r} \frac{\dot{\varepsilon}_{v}+\dot{\varepsilon}_{\theta}}{T} \\
& +\int d A \frac{1}{T}\left\{\lambda \nabla_{\gamma} T+\left[(-\Pi)_{\gamma \tau}+\sigma_{\gamma \tau}\right] v_{\tau}+\frac{\partial e_{s}}{\partial t}\right\} \\
& +\int d A \frac{1}{T}\left[\left(L \nabla_{\tau} n-\sigma_{\gamma \tau}\right) v_{\tau}-L\left(\frac{\partial n}{\partial t}+v_{\tau} \nabla_{\tau} n\right)\right],
\end{aligned}
$$

in which $\lambda \nabla_{\gamma} T+\left[(-\Pi)_{\gamma \tau}+\sigma_{\gamma \tau}\right] v_{\tau}+\partial e_{s} / \partial t$ is the energy flux into the fluid across the fluid-solid interface, and $\left(L \nabla_{\tau} n-\sigma_{\gamma \tau}\right) v_{\tau}-L\left(\partial n / \partial t+v_{\tau} \nabla_{\tau} n\right)$ is the rate of heat production per unit area at the solid surface. We hereby assume that $\left(L \nabla_{\tau} n-\sigma_{\gamma \tau}\right) v_{\tau}$ is positive definite and quadratic in $v_{\tau}$, and $-L\left(\partial n / \partial t+v_{\tau} \nabla_{\tau} n\right)$ is also positive definite and quadratic in $\partial n / \partial t+v_{\tau} \nabla_{\tau} n$. Accordingly, the two boundary conditions are given by

$$
L \nabla_{\tau} n-\sigma_{\gamma \tau}=\beta v_{\tau}
$$

with $\beta$ being the slip coefficient, and

$$
-L=\alpha\left(\frac{\partial n}{\partial t}+v_{\tau} \nabla_{\tau} n\right)
$$

with $\alpha$ being a damping coefficient. They lead to the rate of entropy production per unit area at the solid surface

$$
\frac{1}{T}\left[\beta\left(v_{\tau}\right)^{2}+\alpha\left(\frac{\partial n}{\partial t}+v_{\tau} \nabla_{\tau} n\right)^{2}\right] \geq 0 .
$$

Note that we have been working in a reference frame where the solid surface is still and hence $v_{\tau}$ is identical to the (tangential) slip velocity. In a reference frame where the solid wall is moving with velocity $v_{w}$, the slip velocity is defined by $v_{\tau}^{\text {slip }}=v_{\tau}-v_{w}$ and $\left(L \nabla_{\tau} n-\sigma_{\gamma \tau}\right) v_{\tau}^{\text {slip }}$ enters into $d S_{\text {tot }} / d t$ for the rate of heat production due to slip. Accordingly, the slip boundary condition is $L \nabla_{\tau} n-\sigma_{\gamma \tau}=\beta \nu_{\tau}^{\text {slip }}$ and the corresponding rate of heat production per unit area is $\beta\left(v_{\tau}^{\text {slip }}\right)^{2}$.

For the convenience of presenting and interpreting our results, we define and list the following quantities:

$$
\begin{aligned}
& \dot{\varepsilon}_{v s}=\frac{\eta}{2} \sum_{i j}\left(\nabla_{i} v_{j}+\nabla_{j} v_{i}-\frac{2}{3} \delta_{i j} \nabla \cdot \mathbf{v}\right)^{2}, \\
& \dot{\varepsilon}_{v b}=\zeta(\nabla \cdot \mathbf{v})^{2}, \\
& \dot{\varepsilon}_{\theta}=\lambda(\nabla T)^{2} / T, \\
& \dot{\varepsilon}_{\beta}=\beta\left(v_{\tau}^{\text {slip }}\right)^{2}, \\
& \dot{\varepsilon}_{\alpha}=\alpha\left(\partial n / \partial t+v_{\tau} \nabla_{\tau} n\right)^{2},
\end{aligned}
$$

each being the rate of heat production per unit volume or area due to a particular dissipative process. In terms of the above, the rate of change of the total entropy takes the form of

$$
\begin{aligned}
\frac{d S_{\text {tot }}}{d t}= & \int d \mathbf{r} \frac{\dot{\varepsilon}_{v s}+\dot{\varepsilon}_{v b}+\dot{\varepsilon}_{\theta}}{T}+\int d A \frac{1}{T}\left\{\lambda \nabla_{\gamma} T\right. \\
& \left.+\left[(-\Pi)_{\gamma \tau}+\sigma_{\gamma \tau}\right] v_{\tau}+\frac{\partial e_{s}}{\partial t}\right\}+\int d A \frac{\dot{\varepsilon}_{\beta}+\dot{\varepsilon}_{\alpha}}{T} .
\end{aligned}
$$

\section{NUMERICAL SIMULATIONS}

\section{A. Flow geometry}

Numerical simulations were carried out for sheared liquid-gas systems in Couette-flow geometry (see Fig. 1 for a schematic illustration). A two-phase fluid was confined between two planar solid walls parallel to the $x y$ plane, with the two fluid-solid interfaces defined at $z=0$ and $H$. The two fluid phases were arranged with a liquid sandwiched between two gases. The Couette flow was generated by moving the top and bottom walls at a constant speed $V_{0}$ in the $\pm x$ directions, respectively. The surface Helmholtz free energy density

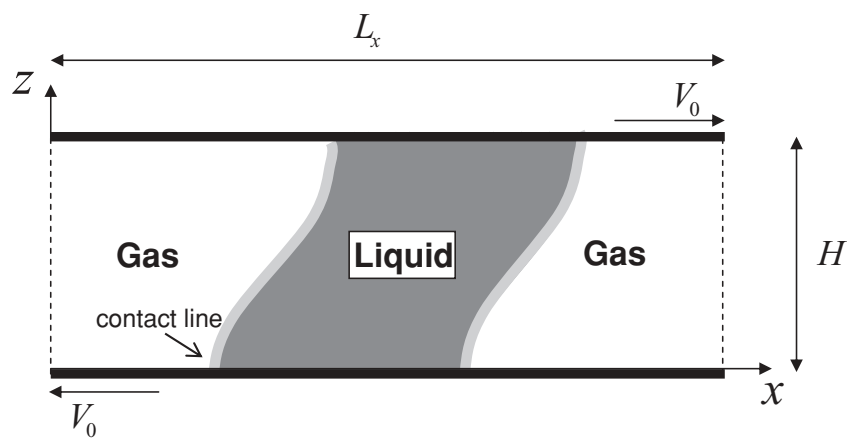

FIG. 1. Schematic illustration for two-phase Couette flows between two planar solid walls. Here the two walls are parallel to the $x y$ plane. The flow is generated by moving the top wall in the $+x$ direction and the bottom wall in the $-x$ direction. With a liquid sandwiched between two gases, two liquidgas interfaces are formed, indicated by the narrow gray regions. 
$f_{s}(n, T)$ was chosen to induce a static contact angle $\theta_{c}=90^{\circ}$, with the two static liquid-gas interfaces being flat and parallel to the $y z$ plane. When sheared by the confining walls, the liquid-gas interfaces become curved in the $x z$ plane. A constant temperature was used at the two fluid-solid interfaces. ${ }^{30}$ The extension in the $x$ direction $L_{x}$ was long enough to allow the stationary single-phase velocity field to appear in gas regions far away from the liquid-gas interfaces, and the system was closed by applying the periodic boundary condition in the $x$ direction.

\section{B. Phenomenological parameters}

A few assumptions have been made for the phenomenological parameters:

(a) $C$ is a (positive) constant and $K$ vanishes, and thus $M=C T$.

(b) The transport coefficients may strongly depend on the density. As proposed by Onuki, ${ }^{30}$ the shear viscosity, bulk viscosity, and thermal conductivity are locally proportional to $n$, with $\eta(n)=\zeta(n)=v_{0} m n$ and $\lambda$ $=k_{B} v_{0} n$, in which $v_{0}=\eta / \rho$ is the kinematic viscosity independent of $n$.

(c) The slip coefficient $\beta$ is a linear function of the number density:

$\beta(n)=\left(\beta_{l}-\beta_{g}\right) \frac{n-n_{g}}{n_{l}-n_{g}}+\beta_{g}$,

with $\beta_{l}\left(\beta_{g}\right)$ being the slip coefficient in the homogeneous liquid (gas) phase and $n_{l}\left(n_{g}\right)$ being the number density of the homogeneous liquid (gas) in liquidgas coexistence equilibrium. The slip length is given by $l_{s}(n)=\eta(n) / \beta(n)$, with $l_{s}\left(n_{l}\right)=\eta\left(n_{l}\right) / \beta_{l}$ being the slip length for liquid and $l_{s}\left(n_{g}\right)=\eta\left(n_{g}\right) / \beta_{g}$ being that for gas. It is further assumed that $l_{s}\left(n_{l}\right)$ and $l_{s}\left(n_{g}\right)$ are equal, with $\beta_{l} / \beta_{g}=n_{l} / n_{g}$.

(d) The damping coefficient $\alpha$ is a constant independent of $n$.

(e) As suggested by Briant et al., ${ }^{27}$ the Helmholtz free energy density at the fluid-solid interface is $f_{s}=-a_{s} n$, with $a_{s}$ being the coupling constant determined by the short-range fluid-solid interaction. Our simulations have confirmed that the static contact angle $\theta_{c}$ at the solid surface can be fixed by $a_{s}$ at a given temperature. In particular, $\theta_{c}=90^{\circ}$ corresponds to $a_{s}=0$.

\section{Dimensionless equations}

To obtain the dimensionless equations suitable for numerical simulations, the length is scaled by $l=\left(C / 2 k_{B} v_{0}\right)^{1 / 2}$, the velocity is scaled by $V_{0}$, the speed of the moving walls in Couette flows, the time is scaled by $l / V_{0}$, the number density $n$ is scaled by $1 / v_{0}$, the total stress $-\stackrel{\leftrightarrow}{\Pi}+\stackrel{\leftrightarrow}{\sigma}$ is scaled by $\varepsilon / v_{0}$, the entropy density $\hat{S}$ is scaled by $k_{B} / v_{0}$, and the temperature $T$ is scaled by $\varepsilon / k_{B}$. The dimensionless continuity equation reads

$$
\frac{\partial n}{\partial t}=-\nabla \cdot(n \mathbf{v})
$$

The Navier-Stokes equation reads

$$
\mathrm{R}\left[\frac{\partial}{\partial t}(n \mathbf{v})+\nabla \cdot(n \mathbf{v v})\right]=\nabla \cdot \overleftrightarrow{M}
$$

in which $\stackrel{\leftrightarrow}{M} \equiv-\stackrel{\leftrightarrow}{\Pi}+\overleftrightarrow{\sigma}$ is the total dimensionless stress, given by

$$
\begin{aligned}
M_{i j}= & -2 T \partial_{i} n \partial_{j} n+\left[-p(n, T)+T(\nabla n)^{2}+2 T n \nabla^{2} n\right] \delta_{i j} \\
& +\mathrm{R} \cdot \operatorname{Re}^{-1} n\left(\partial_{i} v_{j}+\partial_{j} v_{i}+\delta_{i j} \nabla \cdot \mathbf{v} / 3\right),
\end{aligned}
$$

with $p(n, T)=n T /(1-n)-n^{2}$ being the pressure in the equation of state. Here $\mathrm{R}=m V_{0}^{2} / \varepsilon$ is the ratio of the molecular kinetic energy $m V_{0}^{2}$ to the attractive potential energy $\varepsilon$, and $\operatorname{Re}=V_{0} l / \nu_{0}$ appears as a Reynolds number. The dimensionless equation for the entropy density $\hat{S}$ reads

$$
\begin{aligned}
\frac{\partial \hat{S}}{\partial t}= & -\nabla \cdot(\hat{S} \mathbf{v})+\operatorname{Re}^{-1} \nabla \cdot\left(\frac{n \nabla T}{T}\right)+2 \nabla \cdot(n \nabla n \nabla \cdot \mathbf{v}) \\
& +\operatorname{Re}^{-1} \frac{n(\nabla T)^{2}}{T^{2}}+\mathrm{R} \cdot \operatorname{Re}^{-1} \frac{n}{T}\left[2\left(\partial_{x} v_{x}\right)^{2}+2\left(\partial_{z} v_{z}\right)^{2}\right. \\
& \left.+\left(\partial_{z} v_{x}+\partial_{x} v_{z}\right)^{2}+\frac{1}{3}(\nabla \cdot \mathbf{v})^{2}\right] .
\end{aligned}
$$

The dimensionless slip boundary condition reads

$$
n v_{x}^{\text {slip }}=\mathrm{Ls}\left[-n \partial_{\gamma} v_{x}+\mathrm{R}^{-1} \cdot \operatorname{Re}\left(2 T \partial_{\gamma} n-\mathrm{Fs}\right) \partial_{x} n\right],
$$

and the dimensionless equation for the relaxation of $n$ at the solid surface is

$$
\frac{\partial n}{\partial t}+v_{x} \partial_{x} n=-\mathrm{Lb}\left(T \partial_{\gamma} n-\frac{1}{2} \mathrm{Fs}\right) .
$$

Here Ls $=v_{0} m n_{l} / \beta_{l} l$ is the ratio of the liquid slip length $v_{0} m n_{l} / \beta_{l}$ to the length scale $l, \mathrm{Lb}=C \varepsilon / k_{B} \alpha V_{0}$ is inversely proportional to the damping coefficient $\alpha$, and Fs $=a_{s} / \varepsilon l$ measures the strength of fluid-solid coupling. The relation for calculating $T$ from $\hat{S}$ is given by

$$
T=\left[\frac{n}{1-n} \exp \left(\frac{\hat{S}+|\nabla n|^{2}}{n}\right)\right]^{2 / 3} .
$$

It is noted that in the dimensionless equations listed above, there are five dimensionless parameters involved: $\mathrm{R}=m V_{0}^{2} / \varepsilon, \quad \mathrm{Re}=V_{0} l / \nu_{0}, \quad \mathrm{Ls}=v_{0} m n_{l} / \beta_{l} l, \quad \mathrm{Lb}=C \varepsilon /$ $k_{B} \alpha V_{0}$, and Fs $=a_{s} / \varepsilon l$. The numerical results presented in Sec. IV are obtained for $\mathrm{R}=0.0004, \mathrm{Re}=0.1$, and $\mathrm{Fs}=0$. The value of the dimensionless slip length $\mathrm{Ls}=l_{s} / l$ is between 0 and 10 . Note that the length unit $l$ is the characteristic interfacial thickness at temperatures far away from the critical point, and hence is of the same order of magnitude as the molecular diameter. Therefore, the slip length $l_{s}$ used here is of the typical values found in MD simulations. ${ }^{32,33}$ As to the dimensionless rate coefficient $\mathrm{Lb}$, its effects are found to be very weak as long as it is sufficiently large to stabilize the stationary states, and thus Lb $=500$ is used mostly.

The interpretation of our numerical results focuses on the competition among the five different dissipative processes. Five dimensionless rates of entropy production are defined as 
follows:

$$
\begin{aligned}
R_{v s}= & \int d \mathbf{r} \frac{n}{T}\left[2\left(\partial_{x} v_{x}\right)^{2}+2\left(\partial_{z} v_{z}\right)^{2}+\left(\partial_{x} v_{z}+\partial_{z} v_{x}\right)^{2}\right. \\
& \left.-\frac{2}{3}(\nabla \cdot \mathbf{v})^{2}\right] \\
R_{v b} & =\int d \mathbf{r} \frac{n}{T}(\nabla \cdot \mathbf{v})^{2} \\
R_{\theta} & =\mathrm{R}^{-1} \int d \mathbf{r} \frac{n}{T^{2}}(\nabla T)^{2} \\
R_{\beta} & =\mathrm{Ls}^{-1} \int d x \frac{n}{T}\left(v_{x}^{\operatorname{slip}}\right)^{2} \\
R_{\alpha} & =2 \mathrm{Lb}^{-1} \mathrm{R}^{-1} \operatorname{Re} \int d x \frac{1}{T} n^{2}(\nabla \cdot \mathbf{v})^{2}
\end{aligned}
$$

each in the form of $R_{\mathrm{p}}=\int d \mathbf{r} \dot{\varepsilon}_{\mathrm{p}} / T$ or $R_{\mathrm{p}}=\int d x \dot{\varepsilon}_{\mathrm{p}} / T$ scaled by $m v_{0}^{-1} v_{0} V_{0}^{2} k_{B} \varepsilon^{-1}$, with the subscript " $p$ " denoting a particular dissipative process. Note that in our two-dimensional calculations, $\int d \mathbf{r}=\int d x d z$ and $\int d A$ becomes $\int d x$.

In order to concentrate on the hydrodynamics around the liquid-gas interfaces, we introduce a virtual state that serves as a background. It consists of a liquid of density $n_{l}$ and a gas of density $n_{g}$, with the same volume fractions as in the real two-phase state. Assuming no coupling between liquid and gas in the virtual state, we have carried out single-phase Couette-flow simulations for the two phases separately. Entropy production in the single-phase Couette flows is contributed by $R_{v s}$ and $R_{\beta}$ only, and these single-phase rates are subtracted from the corresponding rates calculated for real two-phase flows. An obvious benefit of this subtraction is that the contribution from liquid and/or gas regions far away from the liquid-gas interfaces is removed.

\section{Finite-difference scheme}

As suggested by Teshigawara and Onuki, ${ }^{29}$ the artificial parasitic flow can be avoided if the equation for $\hat{S}$ is used instead of that for $\hat{e}$. We integrated the hydrodynamic Eqs. (3.2), (3.3), and (3.5) with $T=T(n, \nabla n, \hat{S})$ in Eq. (3.8), supplemented with the kinematic boundary condition $v_{\gamma}=0$ and the dynamic boundary conditions (3.6) and (3.7), in a two-dimensional computational domain in the $x z$ plane. A uniform mesh of grid size $0.5 l \times 0.5 l$ was used, with $l=\left(C / 2 k_{B} v_{0}\right)^{1 / 2}$ defined as a length scale for the liquid-gas interfacial thickness (which is $\sim l / \sqrt{1-T / T_{C}}$ for $T$ close to the critical temperature $T_{C}$ ).

We have simulated the two-phase Couette flows confined between two parallel planar solid walls. The variables $n, v_{x}$, $v_{z}, \hat{S}$, and $T$ are defined in an unstaggered, uniformly discretized system. The values of $v_{z}$ and $T$ are fixed at the solid boundaries. The updating of $n$ and $v_{x}$ at the interior sites and the solid boundaries is based on the forward-time centeredspace discretized balance equations, and so is the updating of $v_{z}$ and $\hat{S}$ at the interior sites. Some "ghost" sites of $n$, $v_{x}, v_{z}$, and $T$ are needed in the updating of $n$ and $v_{x}$ at the solid boundaries. The values of $n$ and $v_{x}$ at the ghost sites are determined by the boundary conditions (3.6) and (3.7), respectively. The values of $v_{z}$ and $T$ at the ghost sites are evaluated from the one-sided finite-difference discretized normal derivatives $\partial_{z} v_{z}$ and $\partial_{z} T$ at the solid boundaries. Finally, the temperatures at the interior sites are updated using the centered-space discretization of $T=T(n, \nabla n, \hat{S})$ in Eq. (3.8). The total mass is well conserved, and the scheme is stable for density ratio (of liquid to gas) up to 4.8 , and hence applicable to contact line motion for $T$ as low as $0.875 T_{C}$.

In the limit of infinite thermal conductivity $(\lambda \rightarrow \infty)$, the temperature is homogeneous and equal to the constant value specified at the fluid-solid interfaces. In this limit, there is no need to integrate the entropy equation for determining $T=T(n, \nabla n, \hat{S})$. The results presented in Sec. IV are obtained for homogeneous temperature fields though our algorithm is capable of dealing with finite thermal conductivity. This is to focus on the competition between phase transition and slip as manifested in velocity fields. ${ }^{27}$ The effect of finite thermal conductivity will be discussed in Sec. V.

\section{RESULTS AND DISCUSSION}

\section{A. Two coexisting mechanisms for contact line motion}

In liquid-gas systems, the contact line can move relative to the solid surface through phase transition (evaporation/condensation) even if the no-slip boundary condition is strictly applied. Once the fluid is allowed to slip, however, the contact line can move through both phase transition and slip, with their relative contributions determined by a competition between the rates of entropy production associated with the two coexisting mechanisms.

It has been shown ${ }^{22,23}$ that the continuum hydrodynamic model for contact line motion in two-component fluids can be derived in a variational approach based on the Onsager principle of minimum energy dissipation (entropy production). ${ }^{24,25}$ This principle, as formulated by Onsager for small perturbations away from equilibrium, ${ }^{24,25}$ can also be applied to onecomponent liquid-gas systems in the linear response regime. In fact, this is the regime in which the bulk hydrodynamics is formulated. The boundary conditions can be derived as well through this principle by including the relevant dissipative processes at the fluid-solid interface. Below we outline the application of the Onsager principle to the derivation of the model presented in Sec. II.

For a system described by the variables $\alpha_{1}, \ldots, \alpha_{N}$ measuring the displacement from equilibrium, we need to construct a functional, hereafter denoted by $A$, for minimization with respect to $\dot{\alpha}_{1}, \ldots, \dot{\alpha}_{N}$, the rates of change of the variables $\alpha_{1}, \ldots, \alpha_{N}$. There are two distinct parts in $A=\boldsymbol{\Phi}$ $-\left(\dot{S}+\dot{S}^{*}\right)$. The dissipation function $\boldsymbol{\Phi}=(1 / 2) \sum_{i, j} \eta_{i j} \dot{\alpha}_{i} \dot{\alpha}_{j}$ is half the rate of entropy production, being positive definite and quadratic in the rates $\left\{\dot{\alpha}_{i}\right\}$ with the damping coefficients satisfying the reciprocal relations $\eta_{i j}=\eta_{j i}$. As to the other part $\dot{S}+\dot{S}^{*}, \dot{S}$ is the rate of change of the entropy of the system and $\dot{S}^{*}$ is the rate of the entropy given off to the surroundings. Unlike $\boldsymbol{\Phi}, \dot{S}+\dot{S}^{*}$ is linear in $\left\{\dot{\alpha}_{i}\right\}$, in the form of $\sum_{i} \mathrm{X}_{i} \dot{\alpha}_{i}$ with $\mathrm{X}_{i}$ being the force conjugate 
to the rate $\dot{\alpha}_{i}$. The dissipation function is given by

$$
\begin{aligned}
\boldsymbol{\Phi}= & \int d \mathbf{r}\left[\frac{\eta}{4 T} \sum_{i j}\left(\nabla_{i} v_{j}+\nabla_{j} v_{i}-\frac{2}{3} \delta_{i j} \nabla \cdot \mathbf{v}\right)^{2}\right. \\
& \left.+\frac{\zeta}{2 T}(\nabla \cdot \mathbf{v})^{2}\right]+\int d \mathbf{r}\left[\frac{\mathbf{J}^{2}}{2 \lambda T^{2}}\right] \\
& +\int d A\left[\frac{\beta}{2 T}\left(v_{\tau}^{\text {slip }}\right)^{2}\right]+\int d A\left[\frac{\alpha}{2 T} \dot{n}^{2}\right],
\end{aligned}
$$

with $\mathbf{J}$ denoting the heat current density and $\dot{n}=\partial n / \partial t$ $+v_{\tau} \nabla_{\tau} n$. The rate of increase of the entropy $\dot{S}+\dot{S}^{*}$ is given by

$$
\begin{aligned}
\dot{S}+\dot{S}^{*}= & \int d \mathbf{r}\left[\frac{\stackrel{\leftrightarrow}{\sigma}: \nabla \mathbf{v}}{T}\right]+\int d \mathbf{r}\left[\mathbf{J} \cdot \nabla \frac{1}{T}\right] \\
& +\int d A\left[\frac{1}{T}\left(L \nabla_{\tau} n-\sigma_{\gamma \tau}\right) v_{\tau}^{\text {slip }}\right] \\
& +\int d A\left[-\frac{1}{T} L \dot{n}\right] .
\end{aligned}
$$

The functional $A=\boldsymbol{\Phi}-\left(\dot{S}+\dot{S}^{*}\right)$ is to be minimized with respect to the rates $\{\mathbf{v}, \mathbf{J}, \dot{n}\}$. The Euler-Lagrange (EL) equation with respect to $\mathbf{v}$ gives the expression for the viscous stress $\overleftrightarrow{\sigma}$, the EL equation with respect to $\mathbf{J}$ gives $\mathbf{J}=-\lambda \nabla T$, the EL equation with respect to $v_{\tau}^{\text {slip }}$ at the fluid-solid interface gives the first boundary condition in Eq. (2.22), and the EL equation with respect to $\dot{n}$ at the fluid-solid interface gives the second boundary condition in Eq. (2.23). Physically, these EL equations cover the constitutive equations from the bulk to the interface in the linear response regime. Since $\boldsymbol{\Phi}$ is quadratic in the rates while $\dot{S}+\dot{S}^{*}$ is linear in the rates, we always have $2 \boldsymbol{\Phi}=\dot{S}+\dot{S}^{*}$ for the irreversible processes determined by minimizing $A=\boldsymbol{\Phi}-\left(\dot{S}+\dot{S}^{*}\right)$.

Based on the variational approach outlined above, we can qualitatively deduce some parameter effects on contact line motion, with a focus on the competition between phase transition and slip. As $\boldsymbol{\Phi}$ is positive definite and quadratic in those rates associated with different dissipative processes (five in total), it follows that the magnitude of a particular rate determined from minimizing $A=\boldsymbol{\Phi}-\left(\dot{S}+\dot{S}^{*}\right)$ can be increased by decreasing the corresponding (positive) damping coefficient in $\boldsymbol{\Phi}$. We note that phase transition is measured by $\nabla \cdot \mathbf{v}$, which enters into $\int d \mathbf{r}\left[\zeta(\nabla \cdot \mathbf{v})^{2} / 2 T\right]$ and also $\int d A\left[\alpha \dot{n}^{2} / 2 T\right]$ (with $\dot{n}=-n \nabla \cdot \mathbf{v}$ ) in $\boldsymbol{\Phi}$ while slip is measured by $v_{\tau}^{\text {slip }}$, which enters into $\int d A\left[\beta\left(v_{\tau}^{\text {slip }}\right)^{2} / 2 T\right]$. In what follows, we present a simple analysis with support of numerical results to show the effects of temperature and slip length on the competition between phase transition and slip. For simplicity, we use dimensionless expressions for the various rates of entropy production involved.

In a stationary flow (with $\partial n / \partial t=0$ ), the entropy production associated with phase transition is primarily represented by $R_{v b}$ arising from nonzero $\nabla \cdot \mathbf{v}$, with the converging and diverging velocity fields interpreted as condensation and evaporation, respectively. (In the limit of $\lambda \rightarrow \infty$, the entropy production associated with heat transfer due to latent heat release/absorption vanishes. We will go back to this point in Sec. V.) From $R_{v b}=\int d \mathbf{r} n(\nabla \cdot \mathbf{v})^{2} / T$ and $\mathbf{v} \cdot \nabla n=-n \nabla \cdot \mathbf{v}$, we have $R_{v b}=\int d \mathbf{r}(\mathbf{v} \cdot \nabla n)^{2} / n T$, which indicates that phase transition occurs in the interfacial region penetrated by the flow. It follows that $R_{v b}$ can be approximately expressed as a line integral along the interface (in two-dimensional flows), with the line density given by $\int d \xi v_{\xi}^{2}\left(\partial_{\xi} n\right)^{2} / n T$, where $\int d \xi v_{\xi}^{2}\left(\partial_{\xi} n\right)^{2}$ is an integral along the direction normal to the interface and $v_{\xi}$ is the velocity component normal to the interface, with $\xi$ denoting the spatial coordinate in that direction, which can be relatively well defined if the interfacial thickness is much smaller than the interfacial curvature radius. Since $\int d \xi\left(\partial_{\xi} n\right)^{2}$ scales as $\left(n_{l}-n_{g}\right)^{2} / l_{\text {int }}$ with $l_{\text {int }}$ denoting the interfacial thickness, the quadratic increase of $R_{v b}$ with $v_{\xi}$ slows down as the temperature approaches $T_{C}$. (For $T$ close to $T_{C}$, $\left(n_{l}-n_{g}\right)^{2} / l_{\text {int }} \propto\left(1-T / T_{C}\right)^{3 / 2}$.) Consequently, the interface penetration velocity $v_{\xi}$ is enhanced by the lowered rate of entropy production associated with phase transition. Therefore, the fluid may decrease its slippage at the solid surface while maintaining a moving contact line in a stationary Couette flow between moving walls. This is readily seen from the relation $v_{\xi}+V_{0} \cong v_{x}^{\text {slip }}$ (or $v_{\xi}-V_{0} \cong v_{x}^{\text {slip }}$ ) at the bottom (or top) wall (for dynamic contact angle $\approx 90^{\circ}$ ), in which $\left|v_{\xi}\right|$ and $\left|v_{x}^{\text {slip }}\right|$ always vary in opposite directions between 0 and $V_{0}$. As to the
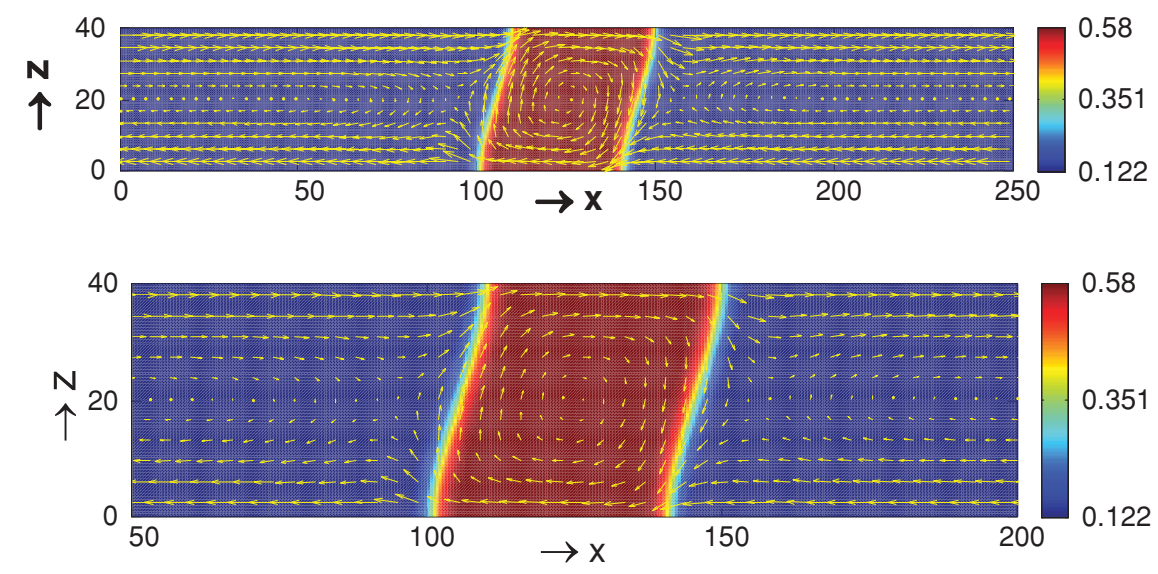

FIG. 2. Density (color) and velocity (arrow) fields for $T=0.875 T_{C}$, Ls $=2$, and $\mathrm{Lb}=500$. The upper panel is for the whole computational domain of $L_{x}=250$ and $H=40$; the lower panel is an enlarged view for the fields near the liquid-gas interfaces. 

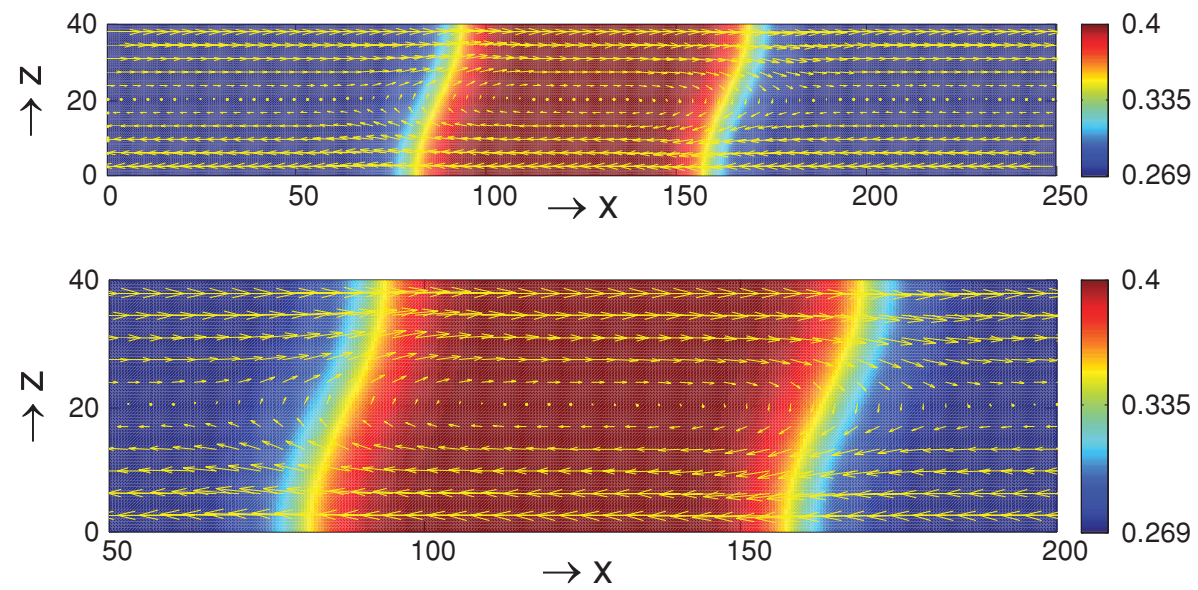

FIG. 3. Density (color) and velocity (arrow) fields for $T=0.99 T_{C}, \mathrm{Ls}=2$, and $\mathrm{Lb}=500$. The upper panel is for the whole computational domain of $L_{x}=250$ and $H=40$; the lower panel is an enlarged view for the fields near the liquid-gas interfaces.

ratio of $\left|v_{\xi}\right|$ to $\left|v_{x}^{\text {slip }}\right|$, it is to be determined by a competition between $R_{v b}$ and $R_{\beta}$ associated with the two mechanisms. In the numerical results presented below, we will tune this competition by changing the temperature and the slip length.

\section{B. Effects of temperature}

We start from $T=0.875 T_{C}$, the lowest temperature with the highest density ratio $n_{l} / n_{g} \approx 4.8$ accessible by our numerical algorithm. Figure 2 shows the density and velocity fields in a stationary Couette flow between two moving walls, with a liquid sandwiched between two gases. The liquid-gas interfaces are significantly penetrated by the flow close to the solid surfaces. It is noted that the interface remains stationary while the fluid is continuously transported across it. This is because phase transition takes place in the interfacial region, exhibited by the converging/diverging velocity fields therein. Away from the solid boundary, the flow tends to be less penetrative.

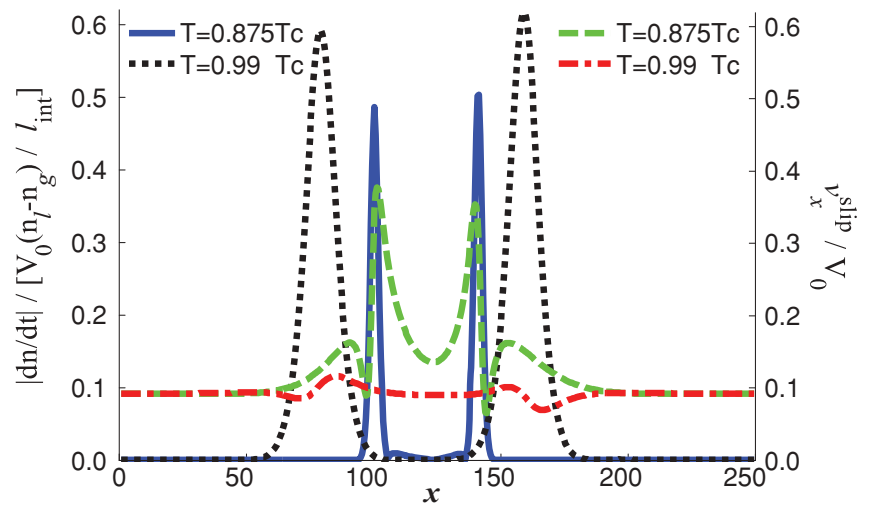

FIG. 4. Variations of $|d n / d t|$ (solid and dotted lines) and $v_{x}^{\text {slip }}$ (dashed and dash-dotted lines) with $x$ at the bottom wall for the two temperatures, computed for $\mathrm{Ls}=2$ and $\mathrm{Lb}=500$. Here $|d n / d t|$ is measured by $V_{0}\left(n_{l}-n_{g}\right) / l_{\text {int }}$, with $l_{\text {int }}$ being the liquid-gas interfacial thickness, and $v_{x}^{\text {slip }}$ is measured by $V_{0}$, the wall speed. Defined as the distance for $n$ to vary from $\left(3 n_{g}+n_{l}\right) / 4$ to $\left(n_{g}+3 n_{l}\right) / 4$ in equilibrium, $l_{\text {int }}$ is found to be $2.5 l$ for $T=0.875 T_{C}$ and $8.5 l$ for $T=0.99 T_{C}$.
We then turn to another temperature $T=0.99 T_{C}$ very close to the critical point. Figure 3 shows the density and velocity fields in the corresponding Couette flow. Compared to Fig. 2, the interface is widened and penetrated by a larger flux of fluid. That is, the interface is now stabilized by faster phase transition within. Correspondingly, the amount of fluid slip at solid surfaces is reduced. As described in Sec. IV A, a moving contact line is maintained in a stationary Couette flow by both phase transition and slip, with their relative weights determined by a competition between the rates of entropy production associated with the two mechanisms. With $T$ getting closer to $T_{C}$, the increase of $R_{v b}$ with the interface penetration velocity is slowed down by the increased interfacial thickness and the decreased density difference $n_{l}-n_{g}$. As a consequence, the lowered rate of entropy production $R_{v b}$ makes the system rely more on phase transition, increasing the fluid flux through the interface and hence decreasing the fluid slip at solid surfaces.

Figure 4 shows the variations of $|d n / d t|=\left|v_{x} \partial_{x} n\right|$ at the bottom wall for the two temperatures. Here $|d n / d t|$ is measured by $V_{0}\left(n_{l}-n_{g}\right) / l_{\text {int }}$, an absolute scale of $v_{x} \partial_{x} n$. It is readily seen that at $T=0.99 T_{C}$, phase transition is faster and distributed in a wider region. The variations of the slip

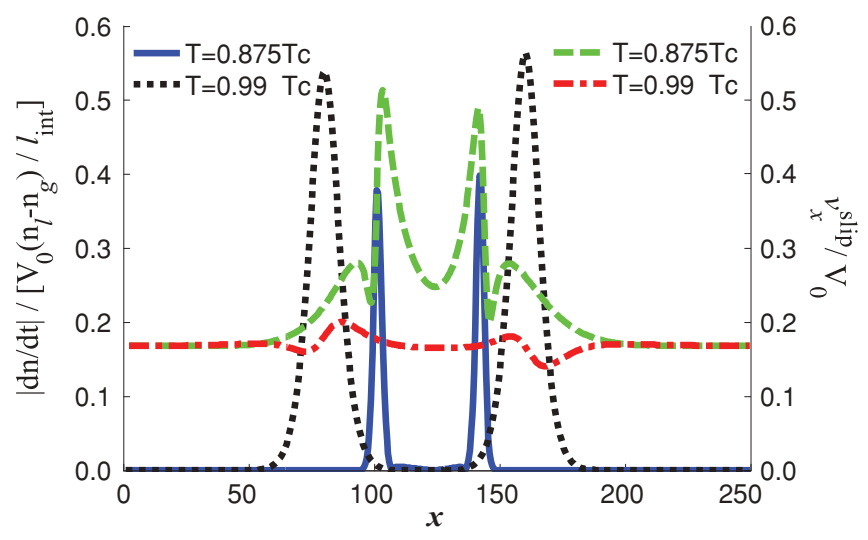

FIG. 5. Variations of $|d n / d t|$ (solid and dotted lines) and $v_{x}^{\text {slip }}$ (dashed and dash-dotted lines) with $x$ at the bottom wall for the two temperatures. Compared to Fig. 4, only the slip length is changed to Ls $=4$. 


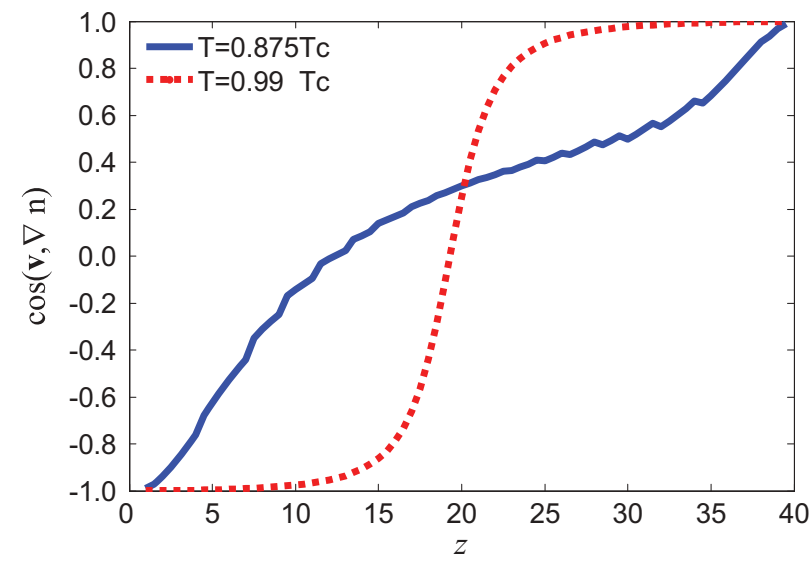

FIG. 6. Variations of $\cos (\mathbf{v}, \nabla n)$ with $z$ along the interface from the bottom to the top wall, computed for $\mathrm{Ls}=2$ and $\mathrm{Lb}=500$. Here $\cos (\mathbf{v}, \nabla n)$ denotes the cosine of the angle between $\mathbf{v}$ and $\nabla n$ evaluated at the level curve of $n(x, z)=\left(n_{l}+n_{g}\right) / 2$.

velocity at the bottom wall are also depicted in Fig. 4, showing that the faster phase transition is accompanied by the smaller amount of slip. Figure 5 is similar to Fig. 4, with a larger slip length.

The enhanced fluid flux across the interface can also be exhibited by the angle at which the interface is penetrated. Figure 6 shows the variations of $\cos (\mathbf{v}, \nabla n)$ along the interface from the bottom to the top wall, with $\cos (\mathbf{v}, \nabla n)$ denoting the cosine of the angle between $\mathbf{v}$ and $\nabla n$ evaluated at the level curve of $n(x, z)=\left(n_{l}+n_{g}\right) / 2$. It is seen that at $T=0.99 T_{C}$, to a large extent the interface is penetrated at a right angle, with $\mathbf{v}$ parallel or antiparallel to $\nabla n$. That is, the flow is almost not hindered by the presence of interface due to the lowered rate of entropy production $R_{v b}$ therein.

\section{Effects of slip}

As demonstrated by Figs. 4 and 5, the system relies more on slip at lower temperature due to the higher rate of entropy production $R_{v b}$ and hence, relatively, the lower rate of entropy production $R_{\beta}$. The weight of slip can also be increased by

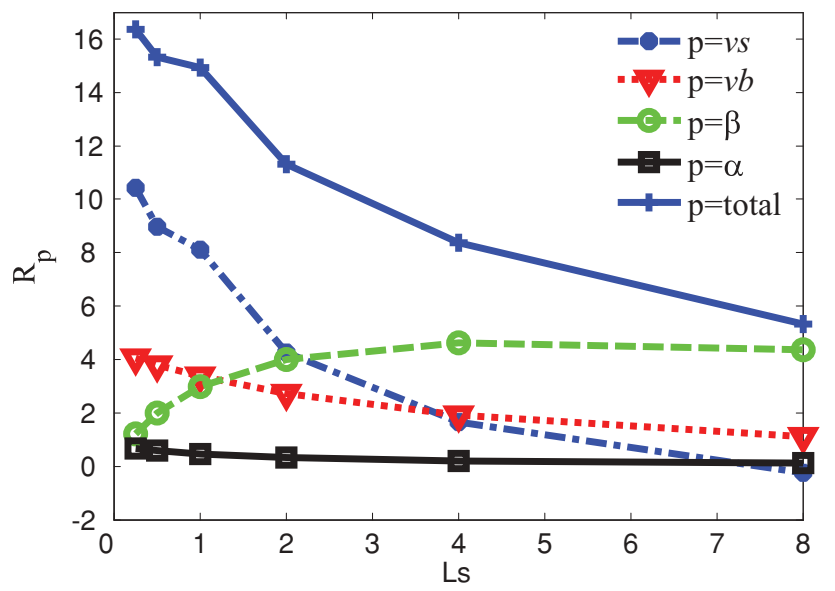

FIG. 7. Variations of the various rates of entropy production (defined in Eq. (3.9), with $R_{\text {total }}=R_{v s}+R_{v b}+R_{\beta}+R_{\alpha}$ ) with the dimensionless slip length $\mathrm{Ls}$ at $T=0.875 T_{C}$, computed using $\mathrm{Lb}=500$.

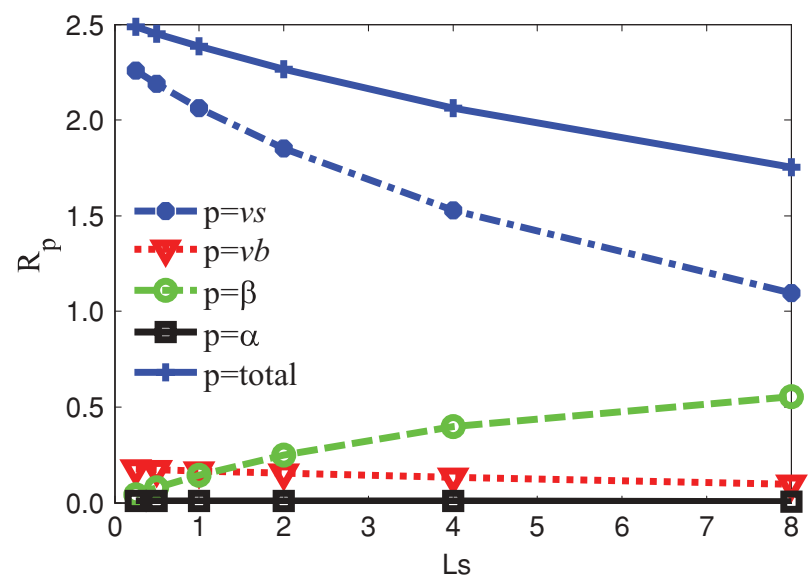

FIG. 8. Variations of the various rates of entropy production (same as in Fig. 7) with the dimensionless slip length Ls at $T=0.99 T_{C}$, computed using $\mathrm{Lb}=500$.

increasing the slip length $l_{s}=\eta / \beta$ and hence lowering $R_{\beta}$. Figures 7 and 8 show the variations of different rates of entropy production with the slip length, for $T=0.875 T_{C}$ and $0.99 T_{C}$, respectively. It is seen that the total rate of entropy production decreases with the increasing slip length. As to the four component parts $R_{v s}, R_{v b}, R_{\beta}$, and $R_{\alpha}$, their variations reveal the competitions among the different dissipative processes. Here the competition is mostly between $R_{v s}$ and $R_{\beta}$, which exhibit appreciable changes in opposite directions. This is particularly clear in Fig. 7, where a larger decrease of $R_{v s}$ is achieved through a smaller increase of $R_{\beta}$. Unlike the classical MCL problem, there is no divergence in $R_{v s}$ at the no-slip limit due to the mass transport across the interface. Nevertheless, a small increase of the slip length from zero (corresponding with no slip) is still able to induce a large decrease of $R_{v s}$. Such a decrease is expected to be even larger if the temperature is further lowered. At $T=0.99 T_{C}$, the effects of an increasing slip length become quantitatively less significant though they remain qualitatively the same. This is simply attributed to the enhanced fluid flux across the interface. That is, as the reliance on slip is reduced, the increase of the slip length becomes less effective in changing $R_{v s}$ and

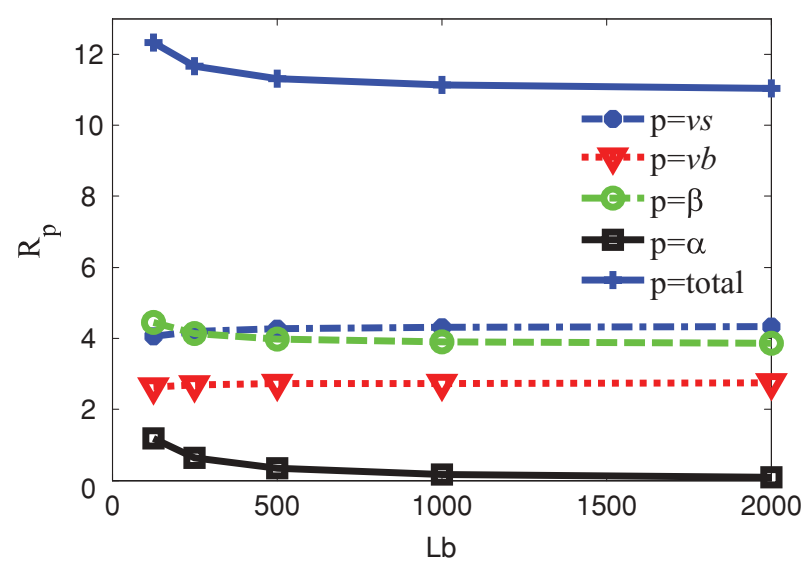

FIG. 9. Variations of the various rates of entropy production (same as in Figs. 7 and 8) with the dimensionless rate coefficient $\mathrm{Lb}$ at $T=0.875 T_{C}$, computed using Ls $=2$. 
$R_{\beta}$, in accordance with the temperature effects discussed in Sec. IV B. Comparing Figs. 7 and 8, we see that $R_{v b}$ for Ls $\rightarrow 0$ is relatively much smaller at $T=0.99 T_{C}$, showing phase transition to be the dominant mechanism in stabilizing the moving contact lines. Therefore, the system only seeks little help from slip and is not so sensitive to the variation of slip length.

\section{Other boundary effects}

The effects of $\alpha$ in the second boundary condition for the relaxation of $n$ at the solid surface are illustrated by Fig. 9 . Not surprisingly, it is $R_{\alpha}$ that shows the largest variation with $\mathrm{Lb} \propto 1 / \alpha$. Physically, although an increasing Lb (i.e., a decreasing $\alpha$ ) tends to enhance $\dot{n}=\partial n / \partial t+v_{\tau} \partial_{\tau} n$ at the solid surface, this effect is actually very limited because $\dot{n}$ at the surface is correlated with that in the bulk and the latter tends to remain unchanged. Consequently, $R_{\alpha}$ varies with $\mathrm{Lb}$ as $R_{\alpha} \propto 1 / \mathrm{Lb}$ approximately. Numerically, $\dot{n}$ is found to be suppressed a little bit when Lb becomes sufficiently small. This is simply to reduce $R_{\alpha} \sim \dot{n}^{2} / \mathrm{Lb}$. (It is also found that a stationary state can no longer be stabilized if Lb is too small. Here it is noted that assuming $L=0$ at the fluid-solid interface ${ }^{30}$ corresponds to $\alpha=0$ and $\mathrm{Lb}=\infty$.) A suppressed $\dot{n}$ leads to a weak deviation from $R_{\alpha} \propto 1 / \mathrm{Lb}$ and makes $R_{v b}$ decrease slightly with the decreasing Lb. Correspondingly, the slippage has to increase slightly with the decreasing $\mathrm{Lb}$, as seen from the variation of $R_{\beta}$. It is also noted that $R_{v s}$ and $R_{\beta}$ still vary in opposite directions, though in a much weaker way compared to Figs. 7 and 8. Overall, we find the effects of $\mathrm{Lb}$ are very weak as long as it is large enough for the stationary state to be stabilized. In this regard, it is a good approximation to use $L=0$ [Eq. (2.9)] under the assumption of fast relaxation (i.e., $\alpha \rightarrow 0$ ) toward equilibrium at the solid surface.

\section{CONCLUSION AND REMARKS}

In this paper, the boundary conditions for the dynamic van der Waals theory have been derived by introducing two additional dissipative processes at the fluid-solid interface. Numerical simulations have been carried out for two-phase Couette flows confined between two planar solid walls with a constant shear rate $2 V_{0} / H$. For slow flows (i.e., small perturbations away from equilibrium), the rate of energy dissipation should be quadratic in the shear rate. It follows that the driving force maintaining each moving wall is proportional to the shear rate. The proportionality constant in this linear relation can be regarded as a damping coefficient. Based on the Onsager principle of minimum energy dissipation, this damping coefficient is determined by the competition among all the different dissipative processes. According to the discussion in Sec. IV A, the variational nature of the Onsager principle states that the system will rely more on a dissipative process if the damping coefficient particular to that process is reduced. For the contact line, it can move through both phase transition and slip, with their relative contributions determined by a competition between the two coexisting mechanisms. At temperatures very close to the critical temperature, the phase transition is dominant, for the liquid-gas interface is wide and the density ratio is close to one. At low temperatures, the slip effect shows up with the increasing slip length.

We would like to point out that all the numerical results presented here are obtained for isothermal systems, i.e., systems with infinite heat conductivity $\lambda$ and homogeneous temperature $T$. It is worth emphasizing that even in these isothermal systems, heat current density $\mathbf{J}=-\lambda \nabla T$ is still nonzero and determined by $\nabla \cdot \mathbf{J}=-\partial \hat{e} / \partial t-\nabla \cdot(\hat{e} \mathbf{v})-\stackrel{\leftrightarrow}{\Pi}$ : $\nabla \mathbf{v}+\dot{\varepsilon}_{v}$ from the equation for $\hat{e}$. Physically, it is the heat current that carries the heat from latent heat release/absorption in the liquid-gas transition. However, for $\lambda \rightarrow \infty$, the entropy production $\int d \mathbf{r} \mathbf{J}^{2} / \lambda T^{2}$ due to heat conduction approaches zero. As a consequence, the rate of entropy production associated with phase transition is represented by $\int d \mathbf{r} \zeta(\nabla \cdot \mathbf{v})^{2} / 2 T$ only and thus underestimated. According to our theoretical interpretation based on the variational formulation of the problem, this will make the liquid-gas transition more preferred (in competition with slip) than in systems with finite heat conductivity. In fact, numerical results have already been obtained for inhomogeneous temperature fields with finite $\lambda$, showing that the amount of slip indeed becomes larger compared with that obtained for infinite $\lambda$.

We also would like to point out that all the fluctuations have been neglected in the present diffuse-interface hydrodynamic model. Physically, not only can the liquid-gas transition be realized via converging/diverging velocity fields, but it can also be realized through fluctuation-assisted nucleation. In particular, nucleation events become more probable as the critical temperature is approached and hence the free energy barrier is reduced. To incorporate the fluctuation effects into a more general hydrodynamic model represents a future work to do.

In the diffuse-interface treatments, ${ }^{31}$ the order parameter is the density $n$ for one-component fluids or the composition $\varphi$ for binary fluids. It is interesting to note that, if $n$ is replaced by $\varphi$, then the boundary conditions derived for liquid-gas systems become formally identical to those derived for binary fluids. ${ }^{20-23}$ This formal resemblance may be attributed to the force balance in the tangential direction at the solid surface, which involves the following four forces in both two types of systems: (i) the $-\gamma \tau$ component of the viscous stress tensor, (ii) the $-\gamma \tau$ component of the reversible stress tensor due to the gradient of $n$ (or $\varphi$ ), (iii) the Marangoni force per unit area due to the tangential variation of the surface free energy density, and (iv) the frictional force per unit area by the wall on the fluid, given by $-\beta v_{\tau}^{\text {slip }}$. Based on this resemblance and the same underlying principle, the boundary conditions for liquid-gas systems will be called the GNBC as well.

Finally, we would like to make two remarks on the modeling and effects of boundary slip.

Although the boundary slip is introduced phenomenologically, the phase-field models developed for describing two-phase fluids allow us to bring necessary information into the modeling of slip in the region where the phase field undergoes rapid variation. To the best of our knowledge, this cannot be accomplished by the classical sharp-interface treatments in fluid mechanics. For example, by combining the GNBC with the Cahn-Hilliard hydrodynamic formulation for binary fluids, we have obtained a continuum model which can produce 
velocity fields and interfacial profiles in quantitative agreement with MD results down to the molecular length scale. ${ }^{20,21}$ A phase-field approach has also shown that in a prewetting transition at the solid surface, a large boundary slip can be realized via the formation of a film of gas or phase-separated "lubricant" with lower viscosity between the fluid and the solid wall. ${ }^{34}$

In the absence of evaporation/condensation, analytical and numerical solutions have been obtained for different slip boundary conditions. ${ }^{35,36}$ It has been found that while in the slip region close to the contact line the different slip models exhibit different behaviors, they all exhibit qualitatively the same behavior on the macroscopic length scale. Furthermore, it has been shown that the macroscopic predictions of the different slip models can be quantitatively superimposed if one normalizes the slip length in each model by a constant factor particular to that model. ${ }^{36}$ The above conclusions should be valid for liquid-gas systems as well where the phase transition in the interfacial region provides an additional mechanism for contact line motion. Here we would like to emphasize that the results presented in Sec. IV should be regarded as inner solutions in the vicinity of contact line. Although the specific details of the slip model do not show up on the macroscopic scale, the presence of slip is detectable since it can lower the rate of energy dissipation or even remove the stress singularity. ${ }^{37}$ The slip effect upon the spreading dynamics of a liquid droplet on a solid surface ${ }^{38}$ will be investigated.

\section{ACKNOWLEDGMENTS}

We would like to thank Professor A. Onuki and Mr. Teshigawara for valuable discussions. We also would like to thank Professor X. Wang for his help on numerical computation. This publication is based on work partially supported by Award No. SA-C0040/UK-C0016, made by King Abdullah University of Science and Technology (KAUST), Hong Kong RGC Grant Nos. 602007 and 603510.
${ }^{1}$ G. K. Batchelor, An Introduction to Fluid Dynamics (Cambridge University Press, Cambridge, England,1967).

${ }^{2}$ C. Huh and L. E. Scriven, J. Colloid Interface Sci. 35, 85 (1971).

${ }^{3}$ V. E. B. Dussan and S. H. Davis, J. Fluid Mech. 65, 71 (1974).

${ }^{4}$ V. E. B. Dussan, Annu. Rev. Fluid Mech. 11, 371 (1979).

${ }^{5}$ P. G. de Gennes, Rev. Mod. Phys. 57, 827 (1985).

${ }^{6}$ J. Koplik, J. R. Banavar, and J. F. Willemsen, Phys. Rev. Lett. 60, 1282 (1988).

${ }^{7}$ P. A. Thompson and M. O. Robbins, Phys. Rev. Lett. 63, 766 (1989).

${ }^{8}$ T. D. Blake, and J. M. Haynes, J. Colloid Interface Sci. 30, 421 (1969).

${ }^{9}$ L. M. Hocking, J. Fluid Mech. 79, 209 (1977).

${ }^{10}$ C. Huh and S. G. Mason, J. Fluid Mech. 81, 401 (1977).

${ }^{11}$ R. G. Cox, J.Fluid Mech. 168, 169 (1986).

${ }^{12}$ M. Y. Zhou and P. Sheng, Phys. Rev. Lett. 64, 882 (1990).

${ }^{13}$ P. Seppecher, Int. J. Eng. Sci. 34, 977 (1996).

${ }^{14}$ H. Y. Chen, D. Jasnow, and J. Vinals, Phys. Rev. Lett. 85, 1686 (2000).

${ }^{15}$ D. Jacqmin, J. Fluid Mech. 402, 57 (2000).

${ }^{16}$ L. M. Pismen and Y. Pomeau, Phys. Rev. E 62, 2480 (2000).

${ }^{17}$ A. J. Briant and J. M. Yeomans, Phys. Rev. E 69, 031603 (2004).

${ }^{18}$ L. Leger, J. Phys.: Condens. Matter 15, S19 (2003).

${ }^{19}$ C. Neto, D. R. Evans, E. Bonaccurso, H. J. Butt, and V. S. J. Craig, Rep. Prog. Phys. 68, 2859 (2005).

${ }^{20}$ T. Z. Qian, X. P. Wang, and P. Sheng, Phys. Rev. E 68, 016306 (2003).

${ }^{21}$ T. Z. Qian, X. P. Wang, and P. Sheng, Phys. Rev. Lett. 93, 094501 (2004).

${ }^{22}$ T. Z. Qian, X. P. Wang, and P. Sheng, J. Fluid Mech. 564, 333 (2006).

${ }^{23}$ T. Z. Qian, C. Y. Qiu, and P. Sheng, J. Fluid Mech. 611, 333 (2008).

${ }^{24}$ L. Onsager, Phys. Rev. 37, 405 (1931).

${ }^{25}$ L. Onsager, Phys. Rev. 38, 2265 (1931).

${ }^{26}$ W. Ren and W. E, Phys. Fluids 19, 022101 (2007).

${ }^{27}$ A. J. Briant, A. J. Wagner, and J. M. Yeomans, Phys. Rev. E 69, 031602 (2004)

${ }^{28}$ R. Borcia, I. D. Borcia, and M. Bestehorn, Phys. Rev. E 78, 066307 (2008).

${ }^{29}$ R. Teshigawara and A. Onuki, Europhys. Lett. 84, 36003 (2008).

${ }^{30}$ A. Onuki, Phys. Rev. E 75, 036304 (2007).

${ }^{31}$ D. M. Anderson, G. B. McFadden, and A. A. Wheeler, Annu. Rev. Fluid Mech. 30, 139 (1998).

${ }^{32}$ P. A. Thompson and S. M. Troian, Nature 389, 360 (1997).

${ }^{33}$ J.-L. Barrat and L. Bocquet, Phys. Rev. Lett. 82, 4671 (1999).

${ }^{34}$ D. Andrienko, B. Dünweg, and O. I. Vinogradova, J. Chem. Phys. 119, 13106 (2003).

${ }^{35}$ V. E. B. Dussan, J. Fluid Mech. 77, 665 (1976).

${ }^{36}$ P. Sheng and M. Zhou, Phys. Rev. A 45, 5694 (1992).

${ }^{37}$ Y. Pomeau, C. R. Mec. 330, 207 (2002).

${ }^{38}$ R. Teshigawara and A. Onuki, Phys. Rev. E 82, 021603 (2010). 